\title{
Ferromagnetism and Metal-Insulator Transitions in Correlated Electron Systems with Alloy Disorder
}

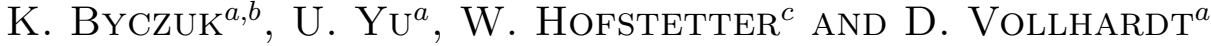 \\ ${ }^{a}$ Theoretical Physics III, Center for Electronic Correlations and Magnetism \\ Institute of Physics, University of Augsburg, 86135 Augsburg, Germany \\ ${ }^{b}$ Institute of Theoretical Physics, University of Warsaw \\ Hoża 69, 00-681 Warszawa, Poland \\ ${ }^{c}$ Institut für Theoretische Physik, Johann Wolfgang Goethe-Universität \\ 60438 Frankfurt/Main, Germany
}

\begin{abstract}
Alloy disorder can affect ferromagnetism and metal-insulator transitions of correlated lattice fermion systems in subtle and often unexpected ways. Solving the Hubbard model and the periodic Anderson model within dynamical mean-field theory we show that alloy disorder can increase the Curie temperature of a non-disordered system, and also yields novel Mott or Kondo insulators at fractional electronic densities.
\end{abstract}

PACS numbers: 71.23.-k, 75.20.Hr, 75.30.Mb

\section{Introduction}

Ferromagnetism and Mott-Hubbard metal-insulator transitions (MIT) in charged lattice fermion systems originate from strong repulsive interactions. Both phenomena occur at intermediate coupling strengths and are notoriously difficult to investigate since they require the application of non-perturbative theoretical methods. Further complications arise in the presence of disorder. Indeed, even in the absence of any interactions the disorder-induced delocalization-localization transition, e.g., caused by a random distribution of two different atoms in an alloy ("alloy disorder"), occurs at a disorder strength comparable to the band width. For this reason disorder also requires a non-perturbative treatment. The simultaneous presence of interactions and disorder therefore leads to a highly non-trivial many-body problem [1-4] which is still far from understood.

In this paper we review our recent investigations of interacting, alloy-disordered lattice fermions. By solving the Hubbard model and the periodic Anderson model (PAM) within the dynamical mean-field theory (DMFT), we show that alloy disorder can lead to:

(i) non-monotonic changes of the Curie temperature $T_{\mathrm{c}}$ as a function of some control parameter, and even to an enhancement of $T_{\mathrm{c}}$ compared to the non-disordered case, and to

(ii) Mott-Hubbard MITs and the formation of the Mott or Kondo insulators at non-integer electron densities.

\section{Alloy-band splitting in the Hubbard model}

As a minimal model describing correlated lattice electrons in the presence of disorder we consider the single-orbital (Anderson-) Hubbard Hamiltonian

$$
H_{\mathrm{H}}=-t \sum_{\langle i j\rangle \sigma} a_{i \sigma}^{\dagger} a_{j \sigma}+\sum_{i \sigma} \epsilon_{i} n_{i \sigma}+U \sum_{i} n_{i \uparrow} n_{i \downarrow},
$$

where $t>0$ is the hopping integral for the electrons between nearest neighbor sites, $U$ is the on-site interaction energy between electrons with opposite spins $\sigma= \pm 1 / 2$, $n_{i \sigma}=a_{i \sigma}^{\dagger} a_{i \sigma}$ is the local electron number operator, and $\epsilon_{i}$ is the local ionic energy which here is a random variable. In the following we assume a bimodal probability distribution for $\epsilon_{i}$, i.e.,

$$
P\left(\epsilon_{i}\right)=x \delta\left(\epsilon_{i}\right)+(1-x) \delta\left(\epsilon_{i}-\Delta\right)
$$

which corresponds to a binary-alloy system composed of two different atoms $A$ and $B$. The atoms are distributed randomly on the lattice and have ionic energies $\epsilon_{A, B}$, with $\epsilon_{B}-\epsilon_{A}=\Delta$. The concentration of $A(B)$ atoms is given by $x=N_{A} / N_{L}\left(1-x=N_{B} / N_{L}\right)$, where $N_{A}\left(N_{B}\right)$ is the number of the corresponding atoms. While the concentration $x$ and energy splitting $\Delta$ are, in general, independent parameters the values $x=0,1$ correspond to a non-disordered system. Hence $\delta=x(1-x) \Delta$ is a natural parameter for the disorder strength of alloy disorder.

From the localization theorem (the HadamardGerschgorin theorem in matrix algebra) it is known that if the Hamiltonian $H_{\mathrm{H}}$ is bounded the single-particle spectrum will open a gap for sufficiently large $\Delta \gg$ 
$\max (|t|, U)[5-7]$. Hence at $\Delta=\Delta_{\mathrm{c}}$ the density of states (DOS) splits into a lower and an upper alloy subbands with centers of mass at the ionic energies $\epsilon_{A}$ and $\epsilon_{B}$, respectively. The width of the alloy gap is of the order of $\Delta$. The lower and upper alloy subbands contain $2 x N_{L}$ and $2(1-x) N_{L}$ states, respectively, where $N_{L}$ is the number of lattice sites.

Our results for the Hubbard model (1) are obtained within the DMFT where, after arithmetic averaging over the disorder using (2), the local one-particle Green function $G_{\sigma}(\tau)=-\left\langle T_{\tau} a_{\sigma}(\tau) a_{\sigma}^{\dagger}(0)\right\rangle$ is given by

$$
G_{\sigma n}=\frac{x}{\mathcal{G}_{\sigma n}^{-1}-\Sigma_{\sigma n}}+\frac{1-x}{\mathcal{G}_{\sigma n}^{-1}-\Delta-\Sigma_{\sigma n}} .
$$

Here we use the finite temperature formalism with odd (fermionic) Matsubara frequencies $\omega_{n}$ indicated by the subscript $n$. The self-energy $\Sigma_{\sigma n}$ is determined by the corresponding impurity problem with the self-consistent mean-field propagator $\mathcal{G}_{\sigma n}=1 /\left(\mathrm{i} \omega_{n}+\mu-\eta_{\sigma n}\right)[8,9]$. The quantity $\eta_{\sigma n}$ is a hybridization function which describes the broadening of the quantum impurity levels due to finite hopping, and $\mu$ is the chemical potential.

In the non-interacting limit $U=0$ the solution (3) corresponds to the result obtained within the coherent potential approximation (CPA) for disordered electrons. In this limit the $\operatorname{DOS} N(\omega)=-\operatorname{Im} G\left(\mathrm{i} \omega_{n}=\omega+\mathrm{i} 0^{+}\right) / \pi$ is found to split into two bands for $\Delta \gg W$, corresponding to alloy subbands with $2 x N_{L}$ and $2(1-x) N_{L}$ states. When the number of electrons $n_{\mathrm{e}} N_{L}$ coincides with the number of available states in the lower alloy subband, the system becomes an alloy band insulator [10] with $n_{\mathrm{e}} \neq 2$ or 4 .

\section{Alloy-band splitting in the periodic Anderson model}

A more complicated situation is encountered in the case of the PAM with binary alloy disorder, given by the Hamiltonian

$$
\begin{array}{r}
H_{\mathrm{PAM}}=\sum_{i, j \sigma} t_{i j} c_{i \sigma}^{\dagger} c_{j \sigma}+\sum_{i \sigma}\left(\varepsilon_{i}^{f} f_{i \sigma}^{\dagger} f_{i \sigma}+\varepsilon_{i}^{c} c_{i \sigma}^{\dagger} c_{i \sigma}\right) \\
+\sum_{i \sigma}\left(V c_{i \sigma}^{\dagger} f_{i \sigma}+V^{*} f_{i \sigma}^{\dagger} c_{i \sigma}\right)+U \sum_{i} n_{i \uparrow}^{f} n_{i \downarrow}^{f}
\end{array}
$$

Here $c_{i \sigma}^{\dagger}\left(c_{i \sigma}\right)$ and $f_{i \sigma}^{\dagger}\left(f_{i \sigma}\right)$ are creation (annihilation) operators of conduction $(c)$ and localized $(f)$ electrons with spin $\sigma$ at a lattice site $i$. The microscopic parameters entering into this model are the hopping amplitude $t_{i j}$ of the $c$-electrons, the random on-site energies $\varepsilon_{i}^{f}$ and $\varepsilon_{i}^{c}$, and $V$, the local hybridization between $f$ - and $c$ electrons. The Coulomb interaction $U$ acts only between $f$-electrons on the same site. The alloy will be modeled by a bimodal probability distribution function,

$$
P\left(y_{i}\right)=x \delta\left(y_{i}-y_{0}\right)+(1-x) \delta\left(y_{i}-y_{0}-\Delta^{y}\right),
$$

where $y_{i}=\varepsilon_{i}^{c}, \varepsilon_{i}^{f}$ are independent, random variables with reference values $y_{0}=\varepsilon_{0}^{c}, \varepsilon_{0}^{f}$. The alloy concentration is characterized by the parameter $x$ and the difference be- tween the atomic energies of the alloy components by $\Delta^{y}=\Delta^{c}, \Delta^{f}$, respectively.

The PAM (4) is solved within the DMFT by mapping it onto a corresponding single-impurity problem. The disordered averaged local Green function is given by the matrix

$$
\begin{aligned}
& \boldsymbol{G}_{\sigma}^{\mathrm{loc}}\left(\tau ;\left\{y_{i}\right\}\right) \\
& \quad=-\left(\begin{array}{ll}
\left\langle T_{\tau} f_{\sigma}(\tau) f_{\sigma}^{\dagger}(0)\right\rangle & \left\langle T_{\tau} f_{\sigma}(\tau) c_{\sigma}^{\dagger}(0)\right\rangle \\
\left\langle T_{\tau} c_{\sigma}(\tau) f_{\sigma}^{\dagger}(0)\right\rangle & \left\langle T_{\tau} c_{\sigma}(\tau) c_{\sigma}^{\dagger}(0)\right\rangle
\end{array}\right)
\end{aligned}
$$

and is expressed in terms of local self-energies, which appears in the $\boldsymbol{k}$-integrated Dyson equation $\boldsymbol{\Sigma}_{\sigma n}=$ $\mathcal{G}_{\sigma n}^{-1}-\boldsymbol{G}_{\sigma n}$. Here $\mathcal{G}_{\sigma n}$ is the local Green function of the non-interacting bath electrons, with

$$
\mathcal{G}_{\sigma n}^{-1}=\left(\begin{array}{cc}
\mathrm{i} \omega_{n}+\mu-\varepsilon_{0}^{f} & V^{*} \\
V & \mathrm{i} \omega_{n}+\mu-\varepsilon_{0}^{c}-\eta_{\sigma n}
\end{array}\right) .
$$

To understand the effect of the disorder on the physics described by the PAM it is instructive to investigate the case $U=0$ first. For $U=0$ the corresponding impurity problem is quadratic and the functional integrals can be performed analytically. However, in the case of a twoband system like the PAM, where $f$ - and $c$-electrons hybridize, the situation is more complicated than in the one-band case discussed earlier, since disorder affects a hybridized two-band system in several nontrivial ways.

We now consider the case where the alloy disorder acts either on the $c$-electrons or the $f$-electrons, respectively. In the case of $c$-electron disorder the diagonal elements of the local Green function are given by

$$
\begin{aligned}
G_{\sigma n}^{c c} & =\frac{x}{\left(\mathcal{G}_{\sigma n}^{c c}\right)^{-1}-|V|^{2} \mathcal{G}_{\sigma n}^{f f}} \\
& +\frac{1-x}{\left(\mathcal{G}_{\sigma n}^{c c}\right)^{-1}-|V|^{2} \mathcal{G}_{\sigma n}^{f f}-\Delta^{c}}, \\
G_{\sigma n}^{f f} & =\frac{x}{\left(\mathcal{G}_{\sigma n}^{f f}\right)^{-1}-|V|^{2} \mathcal{G}_{\sigma n}^{c c}} \\
& +\frac{1-x}{\left(\mathcal{G}_{\sigma n}^{f f}\right)^{-1}-\frac{|V|^{2}}{\left(\mathcal{G}_{\sigma n}^{c c}\right)^{-1}-\Delta^{c}}} .
\end{aligned}
$$

The case of $f$-electron disorder is obtained by exchanging $f \leftrightarrow c$ in (8). Alloy disorder acting only on the $c$-electrons leads to a band splitting of the conduction electrons for large enough energy splitting $\Delta^{\mathrm{c}}$. As in the singleband model each alloy subband then contains $2 x N_{L}$ and $2(1-x) N_{L}$ states, respectively. The $c$-electron alloy subbands are separated by the energy $\Delta^{c}$. One might expect that, due to the hybridization of $c$ - and $f$-electrons, a similar effect would also occur in the $f$-electron subsystem. However, this is not the case. Namely, as seen from (8) a hybridization between the $f$-states and the $2(1-x) N_{L}$ states from the upper alloy $c$-electron subband is no longer possible for $\Delta^{c} \rightarrow \infty$. In this limit a (non-dispersive) $f$-level with $2(1-x) N_{L}$ states appears at the energy $\varepsilon_{0}^{f}$, in analogy with the case without hybridization $(V=0)$. Consequently, for infinitely strong binary alloy disorder in the $c$-electron system $2(1-x) N_{L}$ $f$-electron states become localized for arbitrary but fi- 

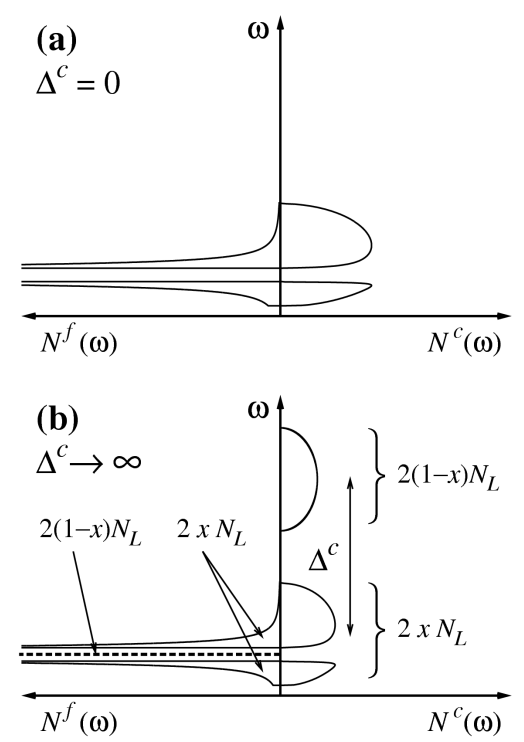

Fig. 1. Binary alloy splitting of the $c$ - and $f$-electron DOS for $U=0$. (a) No disorder in the $c$-electron system $\left(\Delta^{c}=0\right)$. (b) Limit of strong disorder, $\Delta^{c} \rightarrow \infty$; in this case $2(1-x) N_{L}$ non-dispersive $f$-electron states remain at $\varepsilon^{f}$ and $2(1-x) N_{L} \quad c$-electron states are shifted to high energies.

nite values of $V$. So for $\Delta^{c} \rightarrow \infty$ only $2(1-x) N_{L}$ $c$-electron states, rather than $4(1-x) N_{L}$ states, are split off from the spectrum and are shifted to high energies. We note that, although the band splitting scheme is different from the single-band model, the alloy with hybridized $c$ - and $f$-electrons can still be a band insulator for total densities different from integer values (2 or 4 ). A schematic plot in Fig. 1 shows the projected density of states, $N^{b}(\omega)=-\operatorname{Im} \sum_{\sigma} G_{\sigma}^{b b}(\omega) / \pi$, where $b=c$ or $f$, for a system without (a) and with (b) disorder. An analogous analysis of $f$-electron disorder shows that in this case, at large $\Delta^{f}$, the $f$-electron band is split into alloy subbands. Hybridization between the $2(1-x) N_{L}$ states from the upper alloy $f$-band and the $c$-electrons is again prevented when $\Delta^{f} \rightarrow \infty$. Therefore, the corresponding fraction of the $c$-electron band is unchanged, i.e., remains at the same energies as in the non-disordered case. We thus see that even in the absence of interactions binary alloy disorder affects a hybridized two-band system and a single-band system in quite different ways.

\section{Metal-insulator transition at fractional densities}

In the absence of disorder (1) reduces to the usual Hubbard model where a Mott-Hubbard MIT is known to occur upon increasing the interaction strength $U$, provided the number of electrons $N_{\mathrm{e}}$ is commensurate with the number of lattice sites $N_{L}$ (or, more precisely, if the ratio $N_{\mathrm{e}} / N_{L}$ is an odd integer). At zero temperature this is a continuous transition whereas at finite temperatures the transition is of first-order [9]. Surprisingly, in the presence of binary alloy disorder the MIT can occur at fractional densities [10]. We describe this situation by

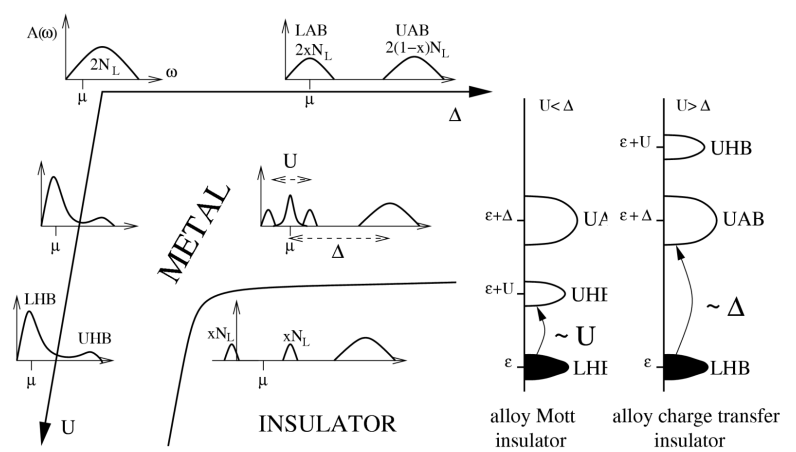

Fig. 2. Left: schematic plot representing the MottHubbard metal-insulator transition in a correlated electron system with the binary alloy disorder. The shapes of the spectral functions $A(\omega)$ are shown for different interactions $U$ and disorder strengths $\Delta$. Increasing $\Delta$ at $U=0$ leads to splitting of the spectral function into the lower (LAB) and the upper (UAB) alloy subbands, which contain $2 x N_{L}$ and $2(1-x) N_{L}$ states, respectively. Increasing $U$ at $\Delta=0$ leads to the occurrence of lower (LHB) and upper (UHB) Hubbard subbands. The Fermi energy for filling $n=x$ is indicated by $\mu$. At $n=x$ (or $n=1+x$, not shown in the plot) the $\mathrm{LAB}(\mathrm{UAB})$ is half-filled. In this case an increase in $U$ and $\Delta$ leads to the opening of a correlation gap at the Fermi level and the system becomes a Mott insulator. Right: two possible insulating states in the correlated electron system with binary-alloy disorder. For $U<\Delta$ the insulating state is an alloy Mott insulator with an excitation gap in the spectrum of order $U$. For $U>\Delta$ the insulating state is an alloy charge transfer insulator with an excitation gap of order $\Delta$; after Ref. [10].
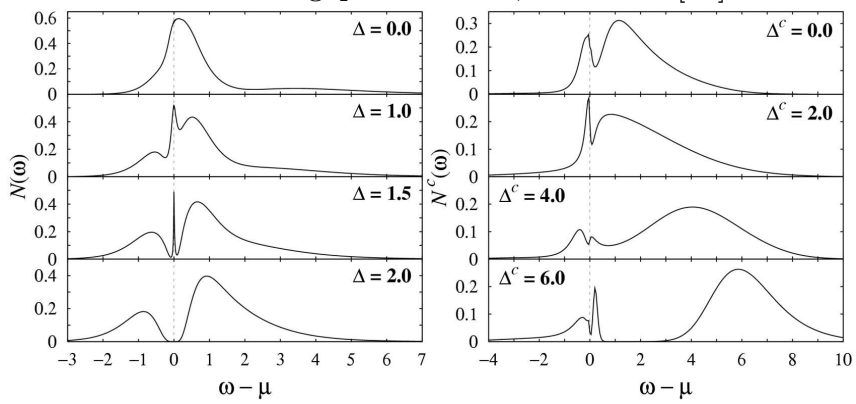

Fig. 3. Left: spectral function of the Hubbard model at $n=x=0.5$ and $U=3$ for different values of the disorder $\Delta$. In the insulating phase $(\Delta=2)$ a Mott gap is opened at the chemical potential $(\omega-\mu=0)$; after Ref. [10]. Right: spectral function of $c$-electrons in the PAM for different $\Delta^{c}$ at $x=0.3$ (other parameters: $U=1.5, V=0.5, \varepsilon_{0}^{c}-\varepsilon_{0}^{f}=3.25$ and $n_{\text {tot }}=1.3$ ) obtained within Quantum Monte Carlo and maximal entropy at $T=1 / 60$. By increasing $\Delta^{c}$ a pseudogap opens, which becomes a real gap for $T \rightarrow 0$; after Ref. [12].

employing the Hubbard model (1) with the disorder distribution (2).

The Mott-Hubbard metal-insulator transition can occur at any density $n=x$ or $1+x$, corresponding to a half-filled lower or upper alloy subband, respectively, as shown schematically for $n=x$ in Fig. 2. The Mott 
insulator can then be approached either by increasing $U$ when $\Delta \geq \Delta_{\mathrm{c}}$ (alloy band splitting limit), or by increasing $\Delta$ when $U \geq U_{\text {c }}$ (Hubbard band splitting limit). The nature of the Mott insulator in the binary alloy system can be understood physically as follows. Due to the high energy cost of order $U$ the randomly distributed ions with lower (higher) local energies $\epsilon_{i}$ are singly occupied at $n=x(n=1+x)$, i.e., the double occupancy is suppressed. In the Mott insulator with $n=x$ the ions with higher local energies are empty and do not contribute to the low-energy processes in the system. Likewise, in the Mott insulator with $n=1+x$ the ions with lower local energies are double occupied implying that the lower alloy subband is blocked and does not play any role. For $U>U_{\mathrm{c}}(\Delta)$, in the Mott insulating state with binary alloy disorder, one may use the lowest excitation energies to distinguish two different types of insulators. Namely, for $U<\Delta$ an excitation must overcome the energy gap between the lower and the upper Hubbard subbands. We call this insulating state an alloy Mott insulator. On the other hand, for $\Delta<U$ an excitation must overcome the energy gap between the lower Hubbard subband and the upper alloy-subband. We call this insulating state an alloy charge transfer insulator. In the left part of Fig. 3 we present the evolution of the DOS for the Hubbard model at filling $n=0.5$ and $U=3$ showing a Mott-Hubbard type MIT. Clearly, there exists a gap at a fractional density of electrons.

A similar opening of a correlation gap, obtained by increasing the alloy band splitting $\Delta^{c}$, is found for the PAM; see right part of Fig. 3. This is caused by the splitting of the $c$-electron band due to binary alloy disorder and the correlations between the $f$-electrons. Namely, when the energy splitting $\Delta^{c}$ is much larger than the width of the $c$-electron band the total number of available low-energy states is reduced from $4 N_{L}$ to $[4-2(1-x)] N_{L}=2(1+x) N_{L}$, whereby the filling effectively increases by a factor of $4 /[2(1+x)]$, such that $n_{\mathrm{tot}}^{\mathrm{eff}}=2 n_{\mathrm{tot}} /(1+x)$, if $n_{\text {tot }}<2(1+x)$. For the filling $n_{\text {tot }}=1.3$ studied in Fig. 3 the concentration $x=0.3$ is a special case since then $n_{\text {tot }}^{\text {eff }}=2$. The system is then effectively at half-filling and behaves as a Kondo insulator at large $U, \Delta^{c}$, and low temperatures. The transition from a metal to a Kondo insulator at non-integer filling predicted here for the PAM is a counterpart to the MottHubbard metal-insulator transition at non-integral filling in the one-band Hubbard model discussed in [10, 11].

\section{Disorder-induced enhancement of the Curie temperature}

Itinerant ferromagnetism in the non-disordered Hubbard model (1) occurs off half-filling and if the DOS is asymmetric and peaked at the lower edge $[13,14]$. While the Curie temperature increases with the strength of the electron interaction one would expect it to be lowered by disorder. However, our investigations show that in some cases the Curie temperature can actually be increased by binary alloy disorder $[11,15]$.

Indeed, the Curie temperature as a function of alloy concentration exhibits very rich and interesting behavior as shown in the left part of Fig. 4. At some concentrations and certain values of $U, \Delta$ and $n$, the Curie temperature is enhanced above the corresponding value for the non-disordered case $(x=0$ or 1$)$. This is shown in the upper left part of Fig. 4 for $0<x<0.2$. The relative increase in $T_{\mathrm{c}}$ can be as large as $25 \%$, as is found for $x \approx 0.1$ at $n=$ $0.7, U=2$ and $\Delta=4$ (upper left part of Fig. 4). This unusual enhancement of $T_{\mathrm{c}}$ is caused by three distinct features of interacting one-band electrons in the presence of binary alloy disorder:

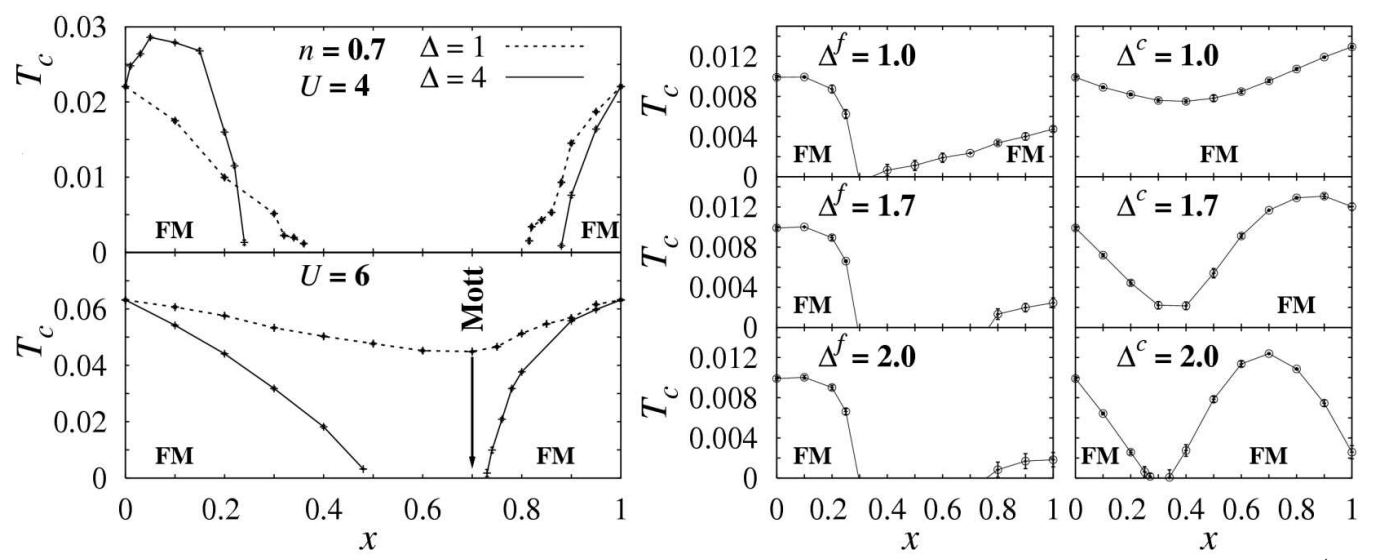

Fig. 4. Left: Curie temperature in the Hubbard model as a function of alloy concentration $x$ at $U=2$ (upper part) and 6 (lower part) for $n=0.7$ and disorder $\Delta=1$ (dashed lines) and 4 (solid lines); after Refs. [11, 15]. Right: Curie temperature in the PAM as a function of alloy concentration $x$ and energy splitting $\Delta^{f}$ (left column) and $\Delta^{c}$ (right column) for $n_{\text {tot }}=1.3$ and $\varepsilon_{0}^{c}-\varepsilon_{0}^{f}=3.25$. Strong $c$-electron disorder enhances $T_{\mathrm{c}}$ compared to its values at $x=0$ or 1; after Ref. [12]. 
(i) The Curie temperature in the non-disordered case, $T_{\mathrm{c}}^{\mathrm{p}} \equiv T_{\mathrm{c}}(\Delta=0)$, depends non-monotonically on the band filling $n$ [13]. Namely, $T_{\mathrm{c}}^{\mathrm{p}}(n)$ has a maximum at some filling $n=n^{*}(U)$, which increases as $U$ is increased; see also the schematic plots in Fig. 5.

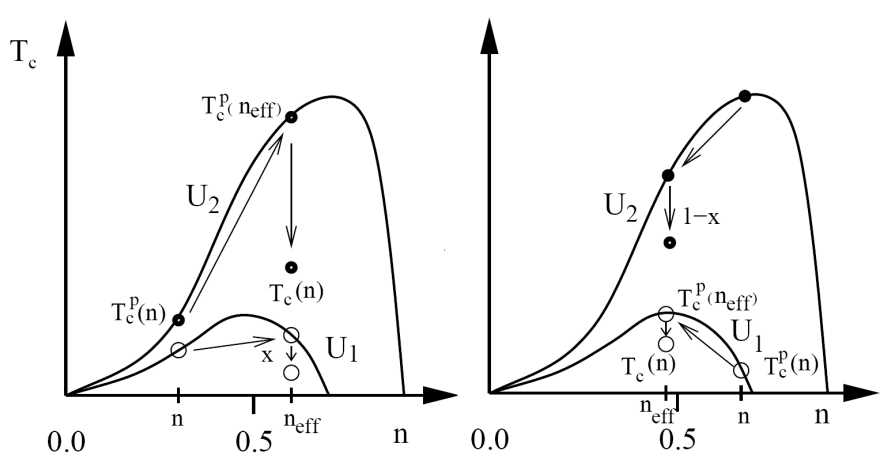

Fig. 5. Schematic plots explaining the filling dependence of $T_{\mathrm{c}}$ for interacting electrons with strong binary alloy disorder. Curves represent $T_{\mathrm{c}}^{\mathrm{p}}$, the Curie temperature for the pure system, as a function of filling $n$ at two different interactions $U_{1} \ll U_{2}$. Left: for $n<x, T_{\text {c }}$ of the disordered system can be obtained by transforming the open (for $U_{1}$ ) and the filled (for $U_{2}$ ) point from $n$ to $n_{\text {eff }}=n / x$, and then multiplying $T_{\mathrm{c}}^{\mathrm{p}}(n / x)$ by $x$ as indicated by arrows. One finds $T_{\mathrm{c}}(n)<T_{\mathrm{c}}^{\mathrm{p}}(n)$ for $U_{1}$, but $T_{\mathrm{c}}(n)>T_{\mathrm{c}}^{\mathrm{p}}(n)$ for $U_{2}$. Right: for $n>x, T_{\mathrm{c}}$ of the disordered system can be obtained by transforming $T_{\mathrm{c}}^{\mathrm{p}}(n)$ from $n$ to $n_{\mathrm{eff}}=(n-2 x) /(1-x)$, and then multiplying $T_{\mathrm{c}}^{\mathrm{p}}[(n-2 x) /(1-x) x]$ by $1-x$ as indicated by arrows. One finds $T_{\mathrm{c}}(n)>T_{\mathrm{c}}^{\mathrm{p}}(n)$ for $U_{1}$, but $T_{\mathrm{c}}(n)<T_{\mathrm{c}}^{\mathrm{p}}(n)$ for $U_{2}$; after Ref. [11, 15].

(ii) As described above, in the alloy-disordered system the band is split when $\Delta \gg W$. As a consequence, for $n<2 x$ and $T \ll \Delta$ electrons occupy only the lower alloy subband, and for $n>2 x$ both the lower and upper alloy subbands are filled. In the former case the upper subband is empty while in the latter case the lower subband is completely full. Effectively, one can therefore describe this system by a Hubbard model mapped either onto the lower or the upper alloy subband, respectively. The second subband plays a passive role. Hence, the situation corresponds to a single band with the effective filling $n_{\text {eff }}=n / x$ for $n<2 x$ and $n_{\text {eff }}=(n-2 x) /(1-x)$ for $n>2 x$. It is then possible to determine $T_{\mathrm{c}}$ from the phase diagram of the Hubbard model without disorder.

(iii) When $\Delta \gg W$ the disorder leads to a reduction of $T_{\mathrm{c}}^{\mathrm{p}}\left(n_{\text {eff }}\right)$, i.e. $T_{\mathrm{c}}(n) \approx \alpha T_{\mathrm{c}}^{\mathrm{p}}\left(n_{\text {eff }}\right)$, where $\alpha=x$ if the Fermi level is in the lower alloy subband and $\alpha=1-x$ if it is in the upper alloy subband. Hence, as illustrated in Fig. $5, T_{\mathrm{c}}$ can be determined by $T_{\mathrm{c}}^{\mathrm{p}}\left(n_{\mathrm{eff}}\right)$. Surprisingly, then, it follows that for suitable values of $U$ and $n$, the Curie temperature of a disordered system can be higher than that of the corresponding non-disordered system (cf. Fig. 5).

As shown in the right part of Fig. 4, the Curie temperature for the transition to the ferromagnetic state in the PAM is therefore a non-monotonic function of the al- loy concentration $x$. In particular, the behavior is quite different for disorder acting on the $f$ - or the $c$-electrons.

\section{1. $f$-electron disorder}

In agreement with Meyer [16] the presence of $f$-electron disorder always reduces the Curie temperature relative to its non-disordered values at $x=0$ or 1 . For strong enough disorder $T_{\mathrm{c}}$ eventually vanishes, e.g., at $x=0.28$ and $x=0.75$, respectively, for $\Delta^{f}=1.7$ (right part, left column of Fig. 4). This is due to the splitting of the $f$-electron band at large $\Delta^{f}$ which increases the double occupation of the lower alloy subband; this reduces the local moment of the $f$-electrons and thereby $T_{\mathrm{c}}$.

\section{2. c-electron disorder}

By contrast, $c$-electron disorder leads to a much more subtle dependence of $T_{\mathrm{c}}$ on concentration $x$. Namely, for increasing energy splitting $\Delta^{c}$ there are, in general, three different features observed, the physical origin of which will be discussed in more detail later:

(i) at $x=1$, i.e., in the non-disordered case, $T_{\mathrm{c}}$ is reduced,

(ii) a minimum develops in $T_{\mathrm{c}}$ at $x=n_{\text {tot }}-1>0$;

(iii) $T_{\mathrm{c}}$ is enhanced over its non-disordered values at $x=0$ or 1 . Altogether this leads to a global maximum in $T_{\mathrm{c}}$ vs. $x$. While the decrease in $T_{\mathrm{c}}$ at $x=1$ is a simple consequence of the reduction of the energy difference between the $f$-level and the $c$-electron band, $\varepsilon^{c}-\varepsilon^{f}=\varepsilon_{0}^{c}-\varepsilon_{0}^{f}-\Delta^{c}$, for increasing $\Delta^{c}$, the latter effects are more subtle.

We now explain the maximum in $T_{\mathrm{c}}$ vs. $x$. It can be understood within the following model based on an ansatz for the Curie temperature, $T_{\mathrm{c}}(U, V, \mu)=$ $T_{\mathrm{c}}^{0}(U, V, \mu) F^{c}\left(\mu-\varepsilon_{0}^{c}\right) F^{f}\left(\mu-\varepsilon_{0}^{f}\right)$, which implies that the formation of local $f$-electron moments $\left(F^{f}\right)$ is assumed to be independent of the $c$-electron mediated ordering of those moments $\left(F^{c}\right)$. In fact, for the Ruderman-KittelKasuya-Yosida (RKKY) model this ansatz can be microscopically justified within a static mean-field theory. The two functions $F^{c}, F^{f}$ are determined by $T_{c}$ calculated within DMFT for the non-disorder case at fixed $\mu-\varepsilon_{0}^{c}$ or $\mu-\varepsilon_{0}^{f}$, respectively; they are shown in Fig. $6 \mathrm{a}$ and $\mathrm{b}$ for one set of parameters. The prefactor $T_{\mathrm{c}}^{0}$ is determined by the requirement that the dimensionless functions $F^{f}$ and $F^{c}$ be equal to one at their maxima. We note that $F^{f}\left(\mu-\varepsilon_{0}^{f}\right)$ has a maximum when the $f$-level is half-filled $\left(\mu=\varepsilon_{0}^{f}+U / 2\right)$, i.e., when the local moment is maximal.

The Curie temperature in the presence of $c$-electron disorder can now be estimated by averaging over the $c$ electron part, $F^{c}$, giving rise to the disorder-dependent function $\mathcal{F}^{c}\left(x, \mu-\varepsilon_{0}^{c}\right)=\left[x F^{c}\left(\mu-\varepsilon_{0}^{c}+\Delta^{c}\right)+(1-\right.$ $\left.x) F^{c}\left(\mu-\varepsilon_{0}^{c}\right)\right]$. The linear dependence on the alloy concentration can again be justified microscopically within a static mean-field theory for the RKKY model, where $T_{\mathrm{c}}$ depends linearly on the DOS at the chemical potential. $T_{\mathrm{c}}$ is now determined for each concentration $x$. 

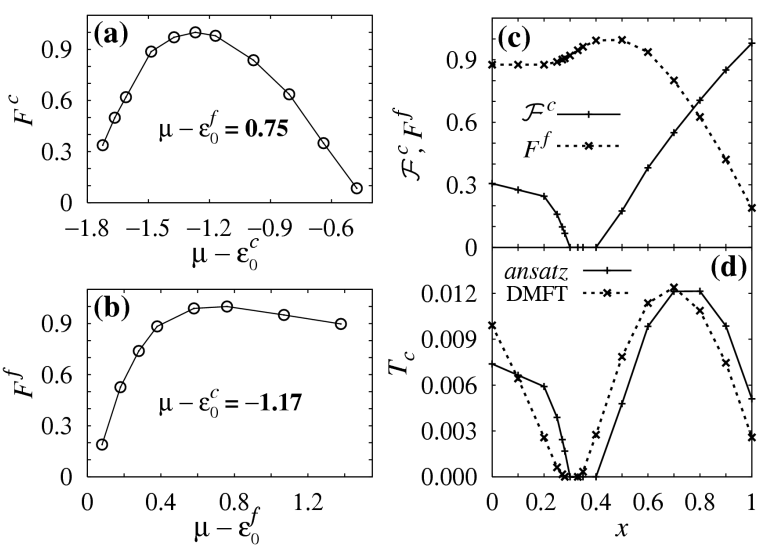

Fig. 6. (a) $F^{c}\left(\mu-\varepsilon_{0}^{c}\right)$, (b) $F^{f}\left(\mu-\varepsilon_{0}^{f}\right)$ appearing in the ansatz for $T_{\mathrm{c}}$ in the disordered PAM (see text) calculated for $\Delta^{c}=0$. (c) $\mathcal{F}^{c}\left(\mu-\varepsilon_{0}^{c}\right)$ and $F^{f}\left(\mu-\varepsilon_{0}^{f}\right)$ for $\Delta^{c}=2.0$; other parameters as in Fig. 4b. (d) Comparison of $T_{\mathrm{c}}$ obtained from the ansatz and within DMFT; after Ref. [12].

We calculate $\mu$, which is an implicit function of $x$, in the non-hybridized limit $(V=0)$ within a rigid band approximation. Th dependence of the resulting functions $\mathcal{F}^{c}\left(x, \mu-\varepsilon_{0}^{c}\right)$ and $F^{f}\left(\mu-\varepsilon_{0}^{f}\right)$ on $x$ are shown in Fig. $6 \mathrm{c}$ for $\Delta^{c}=2.0$. In general, $F^{f}\left(\mu-\varepsilon_{0}^{f}\right)$ has a global maximum at those values of $x$ for which the $f$-level is half-filled (see Fig. 6c). By contrast, $\mathcal{F}^{c}(x, \mu-$ $\left.\varepsilon_{0}^{c}\right)$ is characterized by a wide minimum, related to the formation of the pseudo-gap in the interacting DOS seen in Fig. 3. This minimum reaches zero, i.e., $\mathcal{F}^{c}\left(x, \mu-\varepsilon_{0}^{c}\right)=$ 0 , for a finite range of $x$ values as shown in Fig. 6c. The resulting $T_{\mathrm{c}}(x)$ obtained by the product of these two functions agrees remarkably well with the numerical result obtained by DMFT as shown in Fig. 6d.

\section{Summary}

We showed that the interplay between alloy disorder and electronic correlations can lead to a remarkable increase in the Curie temperature, both in the Hubbard and the periodic Anderson model. Our results provide a basis for further investigation of alloys made of strongly correlated lattice fermions, including electronic ferromagnets such as $\mathrm{Co}_{1-x} \mathrm{Fe}_{x} \mathrm{~S}_{2}$ and $\mathrm{URh}_{1-x} \mathrm{Co}_{x} \mathrm{Ge}$, the Kondo insulator $\mathrm{FeSi}_{1-x} \mathrm{Ge}_{x}$, and ultracold fermionic atoms in optical lattices in the presence of pseudo-random binary disorder, trapped by harmonic potentials.

\section{Acknowledgments}

This work was supported in part by the Sonderforschungsbereich 484 of the Deutsche Forschungsgemeinschaft (DFG).

\section{References}

[1] P.A. Lee, T.V. Ramakrishnan, Rev. Mod. Phys. 57, 287 (1985).

[2] D. Belitz, T.R. Kirkpatrick, Rev. Mod. Phys. 66, 261 (1994).

[3] H. von Löhneysen, Adv. Solid State Phys. 40, 143 (2000).

[4] S.V. Kravchenko, M.P. Sarachik, Rep. Prog. Phys. 67, 1 (2004).

[5] S. Kirkpatrick, B. Velicky̆, H. Ehrenreich, Phys. Rev. B 1, 3250 (1970).

[6] A. Gonis, Green's Functions for Ordered and Disordered Systems, North-Holland, Amsterdam 1992.

[7] We are grateful to B. Velicky for providing us a note with a proof of this fact for non-interacting particles; B. Velicky, private communication, 2001.

[8] D. Vollhardt, Correlated Electron Systems, Vol. 9, Ed. V.J. Emery, World Sci., Singapore 1993, p. 57.

[9] A. Georges, G. Kotliar, W. Krauth, M.J. Rozenberg, Rev. Mod. Phys. 68, 13 (1996).

[10] K. Byczuk, W. Hofstetter, D. Vollhardt, Phys. Rev. B 69, 045112 (2004).

[11] K. Byczuk, M. Ulmke, D. Vollhardt, Phys. Rev. Lett. 90, 196403 (2003).

[12] U. Yu, K. Byczuk, D. Vollhardt, Phys. Rev. Lett. 100, 246401 (2008).

[13] M. Ulmke, Eur. Phys. J. B 1, 301 (1998).

[14] J. Wahle, N. Blümer, J. Schlipf, K. Held, D. Vollhardt, Phys. Rev. B 58, 12749 (1998).

[15] K. Byczuk, M. Ulmke, Eur. Phys. J. B 45, 449 (2005).

[16] D. Meyer, Solid State Commun. 121, 565 (2002). 